\title{
Aspectos da ecologia de Calotropis procera (Apocynaceae) em uma área de Caatinga alterada pelas obras do Projeto de Integração do Rio São Francisco em Mauriti, CE
}

\author{
Aspects of the ecology of Calotropis procera (Apocynaceae) in an area of dry forest altered \\ by works of the Integration Project of the San Francisco River in Mauriti, CE
}

\author{
Juliano Ricardo Fabricante ${ }^{1,2}$, Marina Nabuco Araújo de Oliveira ${ }^{1} \&$ José Alves de Siqueira Filho $^{1}$
}

\begin{abstract}
Resumo
A espécie Calotropis procera (Aiton) W.T. Aiton tem seus primeiros registros no Brasil apontado para 1900. Atualmente ela encontra-se em praticamente todo o País, em especial no Nordeste. Os objetivos do presente estudo foram: (i) aferir a abundância de indivíduos de C. procera; (ii) avaliar aspectos reprodutivos da mesma; (iii) verificar a existência de ação alelopática de seus tecidos; (iv) estimar a área de ocorrência do táxon. A área de estudo encontra-se no município de Mauriti, CE (07²4'07,9”'S e 03842'43,6”W). A média de indivíduos por parcela foi de 11,9 1,9 . A média de frutos por planta foi de $92,20 \pm 33,89$, e a de sementes por fruto foi de $371,26 \pm 4,88$. Os extratos da planta apresentaram ação alelopática. C. procera apresentou ampla probabilidade de ocorrência no Brasil, principalmente na Caatinga, Cerrado e Restinga. Esse conjunto de atributos torna C. procera uma invasora em potencial de áreas de extrema importância para a conservação da biodiversidade brasileira. Palavras-chave: exótica invasora; flor de seda; invasão biológica.
\end{abstract}

\begin{abstract}
The species Calotropis procera (Aiton) WT Aiton has its first records in Brazil pointed to 1900. Currently she is in almost the entire country, particularly in the Northeast. The objectives of this study were: (i) assess the abundance of individuals of C. procera, (ii) evaluate reproductive aspects of the same, (iii) verify the existence of allelopathic action of its tissue, (iv) estimate the area of occurrence of the taxon. The study area is located in the municipality of Mauriti, CE $\left(07^{\circ} 24^{\prime} 07,9^{\prime \prime} \mathrm{S}\right.$ and $\left.038^{\circ} 42^{\prime} 43,6{ }^{\prime \prime} \mathrm{W}\right)$. The average number of individuals per plot was $11.9 \pm 1.9$. The average number of fruits per plant was $92.20 \pm 33.89$, and seeds per fruit was $371.26 \pm 4.88$. The plant extracts showed interfere with germination and growth of other species. C. procera showed wide probability of occurrence in Brazil, mainly in Caatinga, Cerrado and Restinga. This combination of attributes makes $C$. procera a trespasser on potential areas of extreme importance for the conservation of Brazilian biodiversity.

Key words: biological invasion; invasive alien; silk flower.
\end{abstract}

\section{Introdução}

As invasões biológicas constituem uma das maiores ameaças à biodiversidade do planeta (Williamson 1996; Meffe \& Carroll 1997). Contudo, diferentemente de outros problemas ambientais (e.g. fragmentação e queimadas) que podem se atenuar com o tempo, as invasões biológicas tendem a se agravar cada vez mais (Westbrooks 1998). Considera-se exótica invasora todo táxon exótico que se reproduz de forma eficaz mantendo uma população viável, e que é capaz de se dispersar para áreas distantes do local original de introdução e lá estabelecer-se, invadindo a nova região geográfica (Moro et al. 2012).

As exóticas invasoras são altamente eficientes na competição por recursos, podendo vir a causar sérios prejuízos ambientais e econômicos (Daehler 2003). Estima-se que as perdas com as bioinvasões no mundo girem entorno de 1,4 trilhões de dólares anuais, o que representa cerca de $5 \%$ da economia global (SMA 2010).

Devido a esse contexto, e respaldado nas Diretrizes da Resolução CONABIO n ${ }^{\circ} 5$ de 21 de outubro de 2009 que dispõe sobre a estratégia

\footnotetext{
${ }^{1}$ Universidade Federal do Vale do São Francisco - UNIVASF, Centro de Referência para Recuperação de Áreas Degradadas (CRAD), BR 407 km 12 , Lote 543 Projeto Nilo Coelho, s/n C1, 56300-000 Petrolina, PE, Brasil.

${ }^{2}$ Autor para correspondêcia: julianofabricante@hotmail.com
} 
nacional sobre espécies exóticas invasoras (MMA 2009), no tocante as Diretrizes Gerais, 5 (Pesquisa e Monitoramento) e 6 (Educação e Sensibilização Pública), o presente estudo apresenta grande importância, especialmente como subsídio para ações de mitigação relativas à espécie exótica invasora Calotropis procera (Aiton) W.T. Aiton (Apocynaceae).

Introduzida no Brasil por volta de 1900 (Kissmann \& Groth 1992), C. procera pode ser encontrada atualmente em diversas regiões do País (CRIA 2011a; I3N 2011), mas é no Nordeste que estão estabelecidas as grandes populações da espécie (J.A. Siqueira-Filho - com. pess.). Particularmente nas áreas do Eixo Norte do Projeto de Integração do Rio São Francisco com Bacias Hidrográficas do Nordeste Setentrional (PISF) (MIN 2004), C. procera vem ocupando maciçamente centenas de quilômetros de nichos recém-formados pela obra (vide detalhes na descrição do local de estudo).

Assim, os objetivos do presente estudo foram: (i) aferir a abundância de indivíduos de $C$. procera, buscando inferir sobre o atual cenário dessas áreas invadidas pela espécie; (ii) avaliar aspectos reprodutivos da mesma, visando demonstrar a capacidade de dispersão de C. procera; (iii) verificar a existência de ação alelopática de seus tecidos, procurando identificar o poder de exclusão de espécies autóctones por C. procera e; (iv) estimar a área de ocorrência do táxon, buscando ponderar sobre o potencial de invasão de $C$. procera no Nordeste e no Brasil.

\section{Material e Métodos}

Área de estudo

Para a construção de um dos trechos do Eixo Norte do PISF (MIN 2004), toda a vegetação de uma área de dezenas de quilômetros de extensão por 100-200 m de largura, foi suprimida há um pouco mais de dois anos. Nesses ambientes ainda foram depositados uma camada de $0,5-1,5 \mathrm{~m}$ de subsolo retirados da perfuração do canal, dando origem a uma paisagem extremamente degradada e homogênea.

Após esses eventos, a área foi abandonada e desde então está sendo colonizada por algumas poucas espécies nativas pioneiras e por C. procera. Um trecho dessa área, com aproximadamente um quilômetro de extensão por $200 \mathrm{~m}$ de largura, no município de Mauriti, CE $\left(07^{\circ} 24^{\prime} 07,9^{\prime \prime}\right.$ S e $038^{\circ} 42^{\prime} 43,6^{\prime \prime} \mathrm{W}, 402 \mathrm{~m}$ de altitude média), foi utilizado para os estudos realizados.
O clima local segundo a classificação de Köppen-Geiger é o BSh (semiárido quente), e os solos predominantes são os Podzólicos VermelhoAmarelo. A vegetação natural é formada pela Savana Estépica (IBGE 1992).

\section{Abundância de Calotropis procera}

A abundância de indivíduos de C. procera na área de estudo foi conhecida utilizou-se o sistema de parcelas (Muller-Dombois \& Ellemberg 1974). Estas apresentavam dimensões de $5 \times 5 \mathrm{~m}$ e foram alocadas aleatoriamente pela área, num total de 10 unidades amostrais.

No interior destas parcelas, todos os indivíduos de C. procera foram contabilizados. Os espécimes ainda foram classificados quanto ao seu estádio ontogenético em adultos e regenerantes. O critério para a hierarquização dos indivíduos foi a presença ou ausência de material reprodutivo (flor e ou fruto), sendo adultos todos que apresentavam pelo menos uma das estruturas e, regenerantes, aqueles sem as mesmas.

\section{Reprodução}

Para a avaliação de aspectos reprodutivos de $C$. procera, foram selecionados aleatoriamente 10 indivíduos adultos em meio à área estudada para a contagem do número de frutos por planta. Ainda foram coletados 100 frutos da espécie (10 de cada indivíduo) para o cômputo do número de propágulos por fruto.

No intuito de aferir sobre o potencial germinativo das sementes de $C$. procera, quatro repetições de 25 sementes, distribuídas em delineamento inteiramente casualizado (DIC), foram acondicionadas sobre papel gerbox em caixa de polietileno transparente e colocadas em câmera de germinação do tipo B.O.D., a uma temperatura constante de $25 \pm 1^{\circ} \mathrm{C}$. O substrato foi mantido em $60 \%$ da capacidade de campo até o final do experimento. A germinação foi avaliada diariamente até a sua estabilização, sendo consideradas germinadas as sementes que apresentavam $2 \mathrm{~mm}$ de protusão radicular (Brasil 2009). Por meio desta contagem, foi conhecido o percentual de germinação das sementes.

\section{Alelopatia}

Buscando verificar o potencial alelopático de C. procera, folhas da espécie foram coletadas e secas em estufa de circulação forçada a uma temperatura de $50^{\circ} \mathrm{C}$, sendo posteriormente 
trituradas e utilizadas para a formulação de extratos. Estes foram compostos pela parte vegetativa da planta, diluída em água destilada. As concentrações utilizadas foram: 0 (testemunha), 5, 10, 15, e 20\% $(\mathrm{p} / \mathrm{v})$. As soluções foram postas em recipientes de vidro envolvidos por papel alumínio para evitar fotodegradação, onde permaneceram em repouso por 24 horas. Após esse período, as soluções foram filtradas e utilizadas nos ensaios.

Para cada concentração dos extratos, utilizou-se dos mesmos procedimentos descrito para o teste de germinação ora mencionado. A espécie utilizada para os testes foi Lactuca sativa L., variedade Mônica SF, com porcentagem de germinação de $98 \%$.

Foram avaliados o percentual de germinação, índice de velocidade de germinação (IVG) e tamanho e matéria seca das plântulas (Labouriau \& Valadares 1976). Os dados foram submetidos à análise de variância e as médias comparadas pelo teste Tukey ( $p \leq 0,05)$ (Tukey 1959). Utilizouse para as análises o software Past versão 1.12 (Hammer et al. 2003).

\section{Áreas suscetíveis a ocorrência de Calotropis procera}

Buscando inferir sobre a área potencial de invasão de $C$. procera no Brasil (probabilidade de ocorrência), foi realizada uma análise de modelagem de nicho ecológico, baseado em variáveis climáticas (precipitação e temperatura). Os pontos de georreferenciamento de ocorrência da espécie foram obtidos nas bases de dados do SpeciesLink (CRIA 2011a) e do Instituto Horus (I3N 2011).

Oito algoritmos foram utilizados para a análise, sendo eles: Bioclim, Climate Space Model, Envelope Score, Environmental Distance, GARP - DesktopGARP implementation, GARP - new openModeller implementation, Maximum Entropy e, SVM (Support Vector Machines). O desempenho de cada algoritmo foi dado pela pontuação Roc. Quanto mais próximo estiver o valor de um (1), melhor o desempenho do modelo (Elith et al. 2006).

Para avaliar o comportamento dos dados climáticos dos locais de ocorrência de C. procera, foram construídos diagramas Boxplot (Cleveland 1994). As informações foram obtidas na planilha gerada pelo programa openModeller versãon 1.1 (CRIA 2011b), utilizado para as modelagens.

\section{Resultados e Discussão}

Abundância de indivíduos de Calotropis procera

A média de indivíduos de $C$. procera por unidade amostral foi de 11,9 $\pm 1,9$ (média \pm desv. padrão em todo o texto), dos quais, $8,3 \pm 2,5$ adultos e 3,5 $\pm 2,1$ regenerantes. Em estudo desenvolvido em uma área de restinga no estado do Rio de Janeiro (Rangel \& Nascimento 2011), em parcelas com a mesma dimensão das utilizadas no presente trabalho, a média de indivíduos por parcela não chegou a 0,5 .

É importante relembrar que o sítio estudado apresenta características especiais devido à deposição de subsolo no local; que a área foi formada a um pouco mais de dois anos; e que nesse curto período de tempo a maioria dos indivíduos de $C$. procera já eram adultos. As condições observadas delegam a $C$. procera atributos importantes como a produção de sementes de fácil dispersão, pioneirismo e maturação precoce de plantas estabelecidas. Estas qualidades são algumas das apontadas como sendo aquelas que permitem que as espécies exóticas se tornem potenciais invasoras (Parker et al. 1999; Alpert et al. 2000; Genovesi 2005; Santana \& Encinas 2008; Bredow 2009).

Apesar de não ter sido objetivo deste estudo, observou-se nas unidades amostrais a ocorrência de apenas outras três espécies (Ipomoea asarifolia (Desr.) Roem. \& Schult. (Convolvulaceae); Jacquemontia sphaerostigma (Cav.) Rusby (Passifloraceae); Turnera subulata Sm.), sendo que todas, apresentavam apenas um indivíduo cada. Duas condições não excludentes devem agir para resultar a baixa riqueza de espécies do local: (i) poucas espécies são capacitadas a se dispersar em ambientes com as características do estudado (primário e recém-formado). Por ser originária de regiões desérticas (Obeid \& Mahmoud 1971), onde as condições edafoclimáticas são estressantes (déficit hídrico, alta amplitude térmica, solos arenosos e pobres em nutrientes), a espécie deve possuir atributos morfofisiológicos que permitam seu estabelecimento e desenvolvimento nesses locais. A habilidade de iniciar o processo de colonização de uma área também foi observada para $C$. procera em campo rupestre ferruginoso em Minas Gerais (Oliveira et al. 2009), (ii) a espécie apresenta a produção de compostos aleloquímicos que inibem o estabelecimento e ou desenvolvimento de outras espécies (Al-Zahrani \& Al-Robai 2007). 


\section{Reprodução}

A média de frutos por planta foi de $92,20 \pm 33,89$, de sementes por fruto foi de $371,26 \pm 4,88$ e a porcentagem de germinação foi de $86 \%$. O número de sementes por fruto foi semelhante aos apresentados por Souto et al. (2008) em um estudo realizado com populações da espécie na Paraíba e, a porcentagem de sementes germinadas ao apontado por Little et al. (1974), sugerindo um padrão independentemente do local.

Utilizando das razões obtidas no presente estudo, uma planta pode produzir em um único momento temporal por volta de 34.230 sementes. Para agravar a situação, soma-se o fato de que a espécie é capaz de florescer e frutificar durante todo o ano (Little et al. 1974; Oliveira et al. 2009). A grande produção de sementes e alta taxa de germinação observadas para a espécie são destacadas como características básicas de espécies potencialmente invasoras (Parker et al. 1999; Genovesi 2005).

\section{Alelopatia}

A porcentagem de sementes germinadas foi sensivelmente reduzida a partir da primeira concentração do extrato, porém, diferenças significativas em relação à testemunha foram verificadas para os extratos com $15 \%$ e $20 \%$ de concentração. Por outro lado, concentrações iguais ou superiores a $5 \%$ já foram suficientes para diminuir a velocidade de germinação, adiando de forma incontestável a germinação das sementes submetidas à presença dos extratos de C. procera (Tab. 1).

O comprimento das plântulas mostrouse fortemente influenciado pelos extratos após a concentração de $5 \%$, chegando a apresentar redução de mais de um quarto (1/4) no seu tamanho. Em relação à matéria seca, diferenças significativas começaram a ser observadas a partir da concentração de $15 \%$ (Tab. 1).

As alterações observadas nos parâmetros de germinação e de desenvolvimento das plântulas nas concentrações mais baixas dos extratos indicam a presença de aleloquímicos nas folhas de $C$. procera. Efeitos semelhantes sobre quatro espécies cultivadas na Arábia Saudita foram observados por Al-Zahrani \& Al-Robai (2007). Os autores concluíram que os compostos alelopáticos existentes nas folhas de $C$. procera desempenham um papel importante na formação de seus habitats, inibindo o crescimento e produção de outras espécies. A liberação de aleloquímicos no ambiente acaba se tornando uma estratégia de interferência competitiva, que pode conferir a espécies com essa capacidade, vantagens sobre outras espécies (Carmo et al. 2007; Albuquerque et al. 2010).

\section{Áreas suscetíveis a ocorrência}

de Calotropis procera

Os algoritmos testados sugerem distribuição semelhante para C. procera no Brasil (Fig. 1). O que é observado é algo análogo ao Arco Pleistocênico de Prado \& Gibbs (1993), com uma "mancha" de ocorrência na porção norte da região amazônica.

Assim, a espécie apresentou alta suscetibilidade de invasão especialmente no chamado "Domínio das Florestas Sazonais Tropicais" (Prado 2003), sobretudo no Cerrado (Savana - IBGE 1992) e Caatinga (Savana Estépica - IBGE 1992). C. procera também revelou alta probabilidade de ocorrência no litoral do Nordeste e Sudeste do Brasil. Porém, deve-se ressaltar que a

Tabela 1 - Porcentagem de germinação (\% Germ.), índice de velocidade de germinação (IVG), comprimento da plântula (CP - cm) e matéria seca da plântula (MS - g) de Lactuca sativa em razão das concentrações dos extratos aquosos de Calotropis procera.

Table 1 - Germination percentage (\% Germ.), Germination speed index (IVG), seedling length (CP - cm) and seedling dry matter (DM - g) of Lactuca sativa because the concentrations of aqueous strata of Calotropis procera.

\begin{tabular}{lccccc}
\hline Parâmetros & \multicolumn{5}{c}{ Concentração dos Estratos* } \\
\hline \% Germ. & $\mathbf{0 \%}$ & $\mathbf{5 \%}$ & $\mathbf{1 0 \%}$ & $\mathbf{1 5 \%}$ & $\mathbf{2 0 \%}$ \\
IVG & $97,75 \pm 0,96 \mathrm{a}$ & $68,5 \pm 29,64 \mathrm{ab}$ & $54,25 \pm 19,21 \mathrm{abc}$ & $29 \pm 25,47 \mathrm{bc}$ & $13,75 \pm 13,25 \mathrm{c}$ \\
CP & $4,929 \pm 0,019 \mathrm{a}$ & $0,212 \pm 0,098 \mathrm{~b}$ & $0,117 \pm 0,097 \mathrm{bc}$ & $0,036 \pm 0,028 \mathrm{c}$ & $0,021 \pm 0,018 \mathrm{c}$ \\
MS & $0,112 \pm 0,033 \mathrm{a}$ & $4,319 \pm 0,797 \mathrm{a}$ & $0,853 \pm 0,274 \mathrm{~b}$ & $1,153 \pm 1,038 \mathrm{~b}$ & $0,68 \pm 0,235 \mathrm{~b}$ \\
\hline
\end{tabular}

* Médias seguidas de mesma letra não diferem entre si a uma probabilidade maior ou igual a $5 \%$ segundo o teste Tukey. 
ocorrência real da espécie, restringe-se as restingas (Formações Pioneiras com influência marinha IBGE 1992).

A espécie é natural de regiões áridas e semiáridas da Ásia e África (Lindley 1985; Rahman \& Wilcock 1991; Chaudhary \& Al-Jowaid 1999) e desenvolve-se em solos de baixa fertilidade, arenosos e ácidos (Sharma 1934; Ferreira 1973; Ferreira \& Gomes 1974). A tolerância a esta combinação de condições edafoclimáticas, certamente faz com que a espécie obtenha sucesso nos ambientes elencados (Savana, Savana Estépica e Formações Pioneiras). Associado a seus atributos, a falta de inimigos naturais na região (Leal et al. 2010) e a degradação antrópica dessas formações vegetacionais, acabam sendo grandes facilitadores para a expansão da área de ocorrência de C. procera, uma vez que a mesma suporta sítios modificados antropogenicamente (Souto et al. 2008).

Frisa-se que as formações citadas apresentam grande importância biológica. Para a Caatinga, estima-se que existam pelo menos 1.012 espécies vegetais, das quais 318 endêmicas (Queiroz 2006), e o Cerrado, é considerado segundo a Organização Conservation International (2011) como uma das áreas brasileiras prioritárias para a conservação
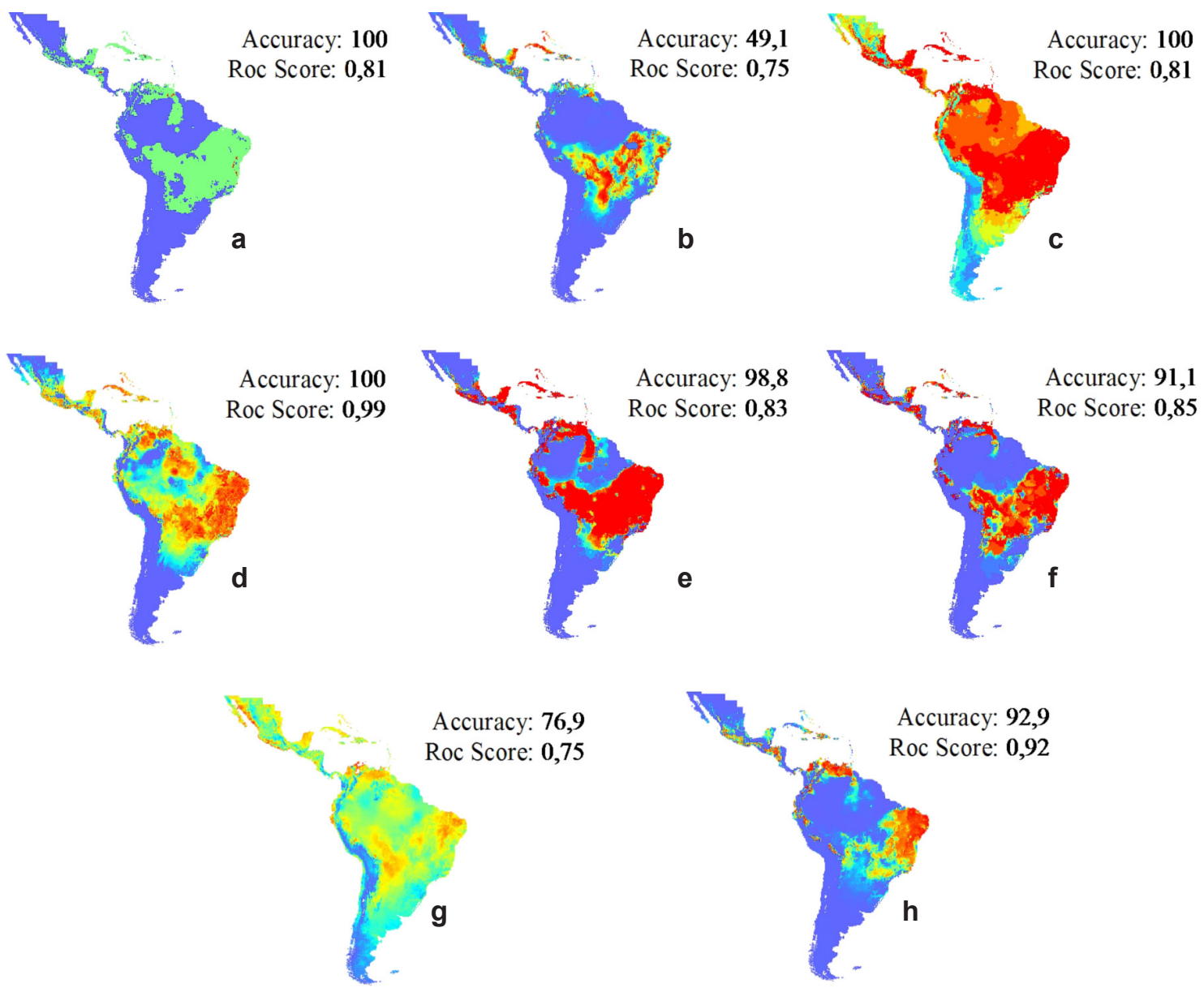

Figura 1 - Probabilidade de ocorrência de Calotropis procera na América do Sul, com os respectivos valores de Precisão (Accuracy) e de Pontuação Roc (Roc Score) para cada algoritmo utilizado. Sendo: a. Bioclim, b. Climate Space Model, c. Envelope Score, d. Environmental Distance, e. GARP - DesktopGARP implementation, f. GARP new OpenModeller implementation, g. Maximum Entropy, h. SVM (Support Vector Machines). Cores frias indicam baixa probabilidade. Cores quentes indicam alta probabilidade.

Figure 1 - Likelihood of Calotropis procera in South America, with corresponding values of Precision (Accuracy) and Score Roc (Roc Score) for each algorithm used. Where: a. Bioclimate, b. Climate Space Model, c. Envelope Score, d. Environmental Distance, e. GARP DesktopGARP Implementation, f. GARP - new openModeller Implementation, g. Maximum Entropy, h. SVM (Support Vector Machines). 
(Hotspot). Já a restinga, apontada como um ecossistema frágil (Hesp 1991) é composta por espécies que ocorrem em áreas com grande diversidade ecológica (Sugiyama 1998).

Conforme a distribuição dos limites de tolerância da espécie à precipitação e a temperatura (Fig. 2), constatou-se que $C$. procera é eurihidrica e estenotérmica. A precipitação no período mais frio variou de 2 a $1074 \mathrm{~mm}$, a precipitação no período mais quente de 44 a $772 \mathrm{~mm}$ e a precipitação total de 585 a $2066 \mathrm{~mm}$. Quanto à temperatura, a média variou de 18 a $27,8^{\circ} \mathrm{C}$, a temperatura no período mais frio de 15,4 a $27^{\circ} \mathrm{C}$, a temperatura no período mais seco de 15,4 a $28,5^{\circ} \mathrm{C}$, a temperatura no período mais quente de 19,9 a $29,1^{\circ} \mathrm{C}$ e, a temperatura no período mais úmido de 19,3 a $27,7^{\circ} \mathrm{C}$.

Uma vez que a espécie possui uma grande amplitude de tolerância à precipitação, a ausência ou baixa ocorrência da mesma no Sul do País está ligada as temperaturas mais amenas que ocorrem naquela região. Porém, precipitações excessivas como as que ocorrem na Mata Atlântica de encosta e parte significativa da Amazônia, também seria outra condição que inibe ou dificulta
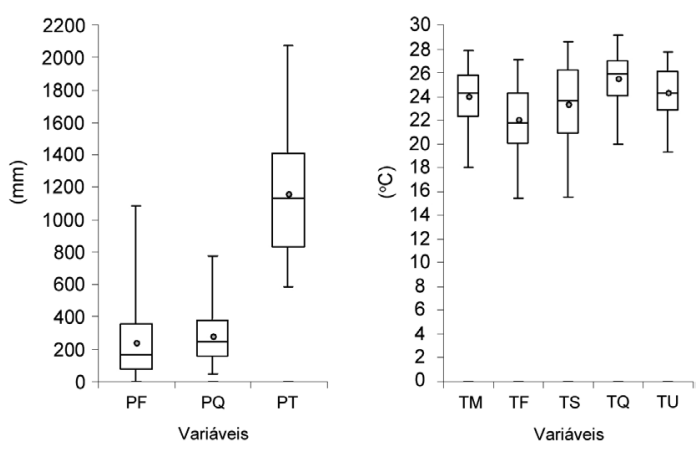

Figura 2 - Boxplot da precipitação (a) e da temperatura (b) dos locais de ocorrência de Calotropis procera. Sendo: $\mathrm{PF}=$ precipitação no período mais frio, $\mathrm{PQ}=$ precipitação no período mais quente, $\mathrm{PT}=$ precipitação total, $\mathrm{TM}=$ temperatura media, $\mathrm{TF}=$ temperatura no período mais frio, TS $=$ temperatura no período mais seco, $\mathrm{TQ}=$ temperatura no período mais quente, $\mathrm{TU}=$ temperatura no período mais úmido.

Figure 2 - Boxplot of precipitation (a) and temperature (b) the places of occurrence of Calotropis procera. Where: $\mathrm{PF}=$ precipitation during the cold, $\mathrm{PQ}=$ precipitation during warmer, $\mathrm{PT}=$ total precipitation, $\mathrm{TM}=$ mean temperature, $\mathrm{TF}=$ temperature during the cold, $\mathrm{TS}=$ temperature at the driest period, $\mathrm{TQ}=$ temperature at the most hot, $\mathrm{TU}=$ temperature in the most humid. o estabelecimento da espécie. Segundo Lindley (1985), C. procera só ocorre em regiões com precipitação superior a $2000 \mathrm{~mm}$ quando os solos do local são excessivamente drenados. Esse fato explicaria porque a espécie ocorre na restinga e não nas formações adjacentes (Mata Atlântica por exemplo) que apresentam as mesmas condições de precipitação.

\section{Conclusão}

A espécie apresentou grande abundância de indivíduos, rápido estabelecimento, produção de grandes quantidades de frutos e sementes, alta taxa de germinação em laboratório, indicativos de substâncias alelopáticas em suas folhas e ampla distribuição geográfica. Esse conjunto de atributos torna $C$. procera uma invasora em potencial de áreas de Caatinga e outras formações vegetacionais brasileiras como o Cerrado e as Restingas.

Devido às características acima listadas não é especulativo afirmar que $C$. procera reflete negativamente sobre a capacidade de resiliência de sítios invadidos, contribuindo assim para a maximização de problemas de degradação ambiental, o que torna urgente e necessária ações de mitigação.

\section{Agradecimentos}

Os autores agradecem ao Ministério da Integração Nacional.

\section{Referências}

Albuquerque, M.B.; Santos, R.C.; Lima, L.M.; Filho, P.A.M.; Nogueira, R.J.M.C.; Câmara, C.A.G. \& Ramos, A.R. 2010. Allelopathy, an alternative tool to improve cropping systems. Agronomy for Sustainable Development 30: 379-395.

Alpert, P.; Bone, E. \& Holzapfel, C. 2000. Invasiveness, invisibility and the role of environmental stress in the spread of non-native plants. Perspectives in Plant Ecology, Evolution and Systematics 3: 52-66.

Al-Zahrani, H.S. \& Al-Robai, S.A. 2007 Allelopathic effect of Calotropis procera leaves extract on seed germination of some plants. JKAU: Sci. 19: 115-126.

Brasil. 2009. Regras para análise de sementes. Ministério da Agricultura, Pecuária e Abastecimento, Brasília. 399p.

Bredow, E.A. \& Wisniewski, C. 2009. Potencial de dispersão de Tecoma stans e atributos químicos de alguns solos do Paraná. Revista Cerne 15: 27-34.

Carmo, F.M.S.; Lima, E.E. \& Borges, E. 2007. Takaki, Massanori. Alelopatia de extratos aquosos de 
canela-sassafrás (Ocotea odorifera (Vell.) Rohwer). Acta Botânica do Brasilica 21: 697-705.

Cleveland, W.S. 1994. The elements of graphing data. $2^{\text {nd }}$ ed. Hobart Press, Summit. 297p.

CRIA. 2011a. SpeciesLink. Disponível em $<$ http://splink. cria.org.br/>. Acesso em 15 Set 2011.

CRIA. 2011b. Openmodeller. Disponível em <http:// openmodeller.cria.org.br/>. Acesso em 20 Set 2011.

Chaudhary, S.A., Al-Jowaid, A.A. 1999. Vegetation of the Kingdom of Saudi Arabia. Ministry of Agriculture e Water, Riyadh. 689p.

Conservation International. 2011. Biodiversyt Hotspots. Disponível em $<$ http://www.biodiversityhotspots. org/Pages/default.aspx>. Acesso em 12 Out 2011.

Daehler, C.C. 2003. Performance comparisons of co-occurring native and alien invasive plants: Implications for Conservation and Restoration. Annual Reviwes 34: 183-211.

Elith. J.; Graham, C.H.; Anderson, R.P.; Dudi'K, M.; Ferrier, S.; Guisan, A.; Hijmans, R.J.; Huettmann, F.; Leathwick, J.R.; Lehmann, A.; Li, J., Lohmann, L.G.; Loiselle, B.A.; Manion, G.; Moritz, C.; Nakamura, M.; Nakazawa, Y.; Overton, J.Mcc.; Peterson, A.T.; Phillips, S.J.; Richardson, K.S.; Scachetti-Pereira, R.; Schapire, R.E.; Sobero, N.J.; Williams, S.; Wisz, M.S. \& Zimmermann, N.E. 2006. Novel methods improve prediction of species' distributions from occurrence data. Ecography 29: 129-151.

Ferreira, M.B. 1973. Distrito Federal e Goiás sob ameaça de invasora Calotropis procera (Ait). R. Br. Revista Cerrados 21: 20-22.

Ferreira, M.B. \& Gomes, V. 1976. Calotropis procera (Ait) R. Br. uma invasora a ser estudada e controlada no Estado de Minas Gerais. Oreades. 5: 68-75.

Genovesi, P. 2005. Eradications of invasive alien species in Europe: a review. Biological Invasions 7: 127-133.

Hammer, O.; Haper, D.A.T. \& Ryan, P.D. 2003. PAST: Paleontological Statistics Software package for education and data analysis. Palaeontologia Electronica, Oslo.

Hesp, P.A. 1991. Ecological processes and plant adaptations on coastal dunes. Journal of Arid Environments 21: 165-191.

I3N. 2011. Consultas de espécies. Disponível em $<$ http:// i3n.institutohorus.org.br/filt_especies.asp $>$. Acesso em 28 Set 2011.

IBGE. 1992. Manual técnico da vegetação brasileira. Rio de Janeiro (Série Manuais Técnicos em Geociências), 1. 92p.

Kismann, K.G., Groth, D. 1992. Plantas infestantes e nocivas. BASF Brasileira, São Paulo. 399p.

Laboriau, L.G., Valadares, M.B. 1976. On the germination of seeds of Calotropis procera. Anais da Academia Brasileira de Ciências 48: 174-186.
Leal, L.C.; Lannuzzi, L. \& Leal, I.R. 2010. Liberação ecológica de espécie invasora: entomofauna associada à Calotropis procera não afeta a germinação de sementes. Revista Nordestina de Zoologia 4: 112-117.

Lindley, J. 1985. Flora medica. Ajay Book Services, New Delhi.

Little, E.L.; Woodbury, R.O. \& Wadsworth, F.H. 1974. Trees of Puerto Rico and the Virgin Islands. Vol. 2. Agriculture Handbook 449. U.S. Department of Agriculture, Washingtion, DC. 1024p.

Meffe, G.K. \& Carroll, C.R. 1997. Principles of conservation biology. $2^{\mathrm{a}}$ ed. Sinawer Associates, Sunderland. $729 \mathrm{p}$.

MIN. 2004. Projeto de Integração do Rio São Francisco com Bacias Hidrográficas do Nordeste Setentrional. Relatório de Impacto Ambiental (RIMA). Ministério da Integração Nacional, Brasília. 136p.

MMA. 2009. Resolução Conabio $n^{\circ} 5$ de 21 de outubro de 2009: Dispõe sobre a estratégia nacional sobre espécies exóticas invasoras. Ministério do Meio Ambiente, Brasília. 27p.

Moro, M.F.; Souza, V.C.; Oliveira-Filho, A.T.; Queiroz, L.P.; Fraga, C.N.; Rodal, M.J.N.; Araújo, F.S. \& Martins, F.R. 2012. Alienígenas na sala: o que fazer com espécies exóticas em trabalhos de taxonomia, florística e fitossociologia? Acta Botanica Brasilica 26: 991-999.

Muller-Dombois, D., Ellemberg, H. 1974. Aims and methods of vegetation ecology. John Wiley \& Sons, New York. 547p.

Obeid, M. \& Mahmoud, A. 1971. Ecological studies in the vegetation of the Sudan II: The ecological relationships of the vegetation of Khartoum Province. Vegetatio 23: 177-198.

Oliveira, S.H.F.; Negreiros, D.; Fernandes, G.W.; Barbosa, N.P.U.; Rocha, R. \& Almeida-Cortez, J.S. 2009. Seedling growth of the invader Calotropis procera in ironstone rupestrian fi eld and seasonally dry forest soils. Neotropical Biology and Conservation 4: 69-76.

Parker, I.M. et al. 1999. Impact: toward a framework for understanding the ecological effects of invaders. Biological Invasions 1: 3-19.

Prado, D.E. \& Gibbs, P.E. 1993. Patterns of species distributions in the dry seasonal forest South America. Annals of the Missouri Botanic Garden 80: 902-927.

Prado, D.E. 2003. As caatingas do Brasil. In: Leal, I.R.; Tabarelli, M. \& Silva, J.M.C. (eds.). Ecologia e conservação da Caatinga. Ed. Universidade Federal de Pernambuco, Recife. Pp. 3-73.

Queiroz, L.P. 2006 The Brazilian caatinga: phytogeographical patterns inferred from distribution data of the Leguminosae. In: Penninigton, R.T.; Lewis, G.P. \& Ratter, J.A. (eds.). Neotropical dry forests and savannas. Royal Botanical Garden. Pp. 113-149. 
Rahman, M.A. \& Wilcock, C.C. 1991. A taxonomic revision of Calotropis (Asclepiadaceae). Nordic Journal of Botany 11: 301-308.

Rangel, E.S. \& Nascimento, M.T. 2011. Ocorrência de Calotropis procera (Ait.) R. Br. (Apocynaceae) como espécie invasora de restinga. Acta Botanica Brasilica. 25: 657-663.

Santana, A.O. \& Encinas, J.I. 2008. Levantamento das espécies exóticas arbóreas e seu impacto nas espécies nativas em áreas adjacentes a depósitos de resíduos domiciliares. Biotemas 21: 29-38.

Sharma, G.K. 1934. Calotropis procera and Calotropis gigantea. Indian Journal Veterinary Science and Animal Husbandry 4: 63-74.

SMA. 2010. Espécies exóticas invasoras. Secretaria de Estado do Meio Ambiente, Coordenadoria de Biodiversidade e Recursos Naturais, Unidade de Coordenação do Projeto de Recuperação das Matas Ciliares, São Paulo. Cadernos da Mata Ciliar 3: 34.

Souto, P.C.; Sales, S.C.V.; Souto, J.S.; Santos, R.V. \& Sousa, A.A. 2008. Biometria de frutos e número de sementes de Calotropis procera (Ait.) R. Br no Semi-árido da Paraíba. Revista Verde. 3: 108-113.

Sugiyama, M. 1998. Estudo de florestas da restinga da Ilha do Cardoso, Cananéia, São Paulo, Brasil. Boletim do Instituto de Botânica 11: 119-159.

Tukey, J.W. 1959. A quick, compact, two-sample test to Duckworth's specications. Technometrics 1: 31 .

Westbrooks, R. 1998. Invasive plants: changing the landscape of America. Fact Book, Washington, DC. 109 p.

Williamson, M. 1996. Biological invasions. Chapman \& Hall, London. 244p. 